\title{
Deciphering the relative contributions of multiple functions within plant-microbe symbioses
}

\author{
Benjamin A. Sikes, ${ }^{1,3}$ Jeff R. Powell, ${ }^{2}$ and Matthias C. Rillig ${ }^{2}$ \\ ${ }^{1}$ Department of Integrative Biology, University of Guelph, Guelph, Ontario N1G 2W1 Canada \\ ${ }^{2}$ Institut für Biologie, Freie Universität Berlin, 14195 Berlin, Germany
}

\begin{abstract}
For microbial symbioses with plants, such as mycorrhizas, we typically quantify either the net effects of one partner on another or a single function a symbiont provides. However, many microbial symbioses provide multiple functions to plants that vary based on the microbial species or functional group, plant species, and environment. Here we quantified the relative contributions of multiple functions provided by arbuscular mycorrhizal (AM) fungi to symbiont-mediated changes in plant biomass. We used two published data sets, one that measured multiple functions (pathogen protection and nutrient uptake) on a single plant and one that measured a single function (pathogen protection) on multiple plants. Using structural equation modeling, we observed strong variation in the functional pathways by which AM fungi altered plant growth; changes in plant biomass were associated with different functions (and different AM fungal functional groups) for the different plant species. Utilizing this methodology across multiple partners and environments will allow researchers to gauge the relative importance of functions they isolate and, perhaps more importantly, those they did not consider. This baseline information is essential for establishing the specific mechanisms by which microbial symbioses influence plant diversity and to more effectively utilize these organisms in agriculture, restoration and conservation.
\end{abstract}

Key words: arbuscular mycorrhizal fungi; microbial symbiosis; multifunctionality; mycorrhizal dependency; net effects; nutrient uptake; pathogen protection.

\section{INTRODUCTION}

Many microbial symbioses with plants are considered multifunctional, with potential benefits to the plant host being derived from multiple mechanisms (mycorrhizas [Newsham et al. 1995, Finlay 2008]; rhizobia [Arora et al. 2001]; grass fungal endophytes [Schardl et al. 2004]; dark-septate endophytes [Mandyam and Jumpponen 2005]). Although multifunctionality is frequently cited for these symbioses, no research has quantified the relative importance of the different functions performed by microbial symbionts to plant fitness or plant community structure. These functions can occur simultaneously, yet certain functions are likely more common or hold greater sway over plant responses. In addition, functional variation among environments and between partners in some plant-microbial symbioses can result in seemingly context-dependent and, at times, idiosyncratic outcomes (Johnson et al. 1997, Klironomos 2003). By simultaneously quantifying the effects of multiple functional pathways, we can establish their relative importance to overall plant response among different partners and environments. Other complex ecological systems have successfully quantified multiple interaction

Manuscript received 14 October 2009; revised 6 January 2010; accepted 19 January 2010. Corresponding Editor: D. A. Wardle.

${ }^{3}$ E-mail: bensikes@gmail.com pathways through analysis of structural equation models (Wootton 1994, Vázquez and Simberloff 2004, Chaudhary et al. 2009) and here we propose to adapt this methodology to the multiple functions of microbial symbioses using mycorrhizal fungi as a model system.

The mutualism formed between plants and arbuscular mycorrhizal (AM) fungi is a ubiquitous microbial symbiosis that can provide multiple functions to plant hosts. AM fungal hyphae extend into the soil surrounding roots. This hyphal network is the mechanism for increased uptake of nutrients and water, as well as changes to soil structure (Smith and Read 2008). The symbiosis is often simplified to plants exchanging photosynthetically derived carbon for increased nutrient uptake by fungal soil hyphae. Soil phosphorus (P) has historically been the focus of AM function and derived plant benefit because it is both scarce and relatively immobile in many ecosystems (Bolan 1991, Smith and Read 2008). Apart from their recognized role in phosphorus uptake, AM fungi can transfer other nutrients $(\mathrm{N}, \mathrm{Zn}, \mathrm{Mg}$, and $\mathrm{Ca})$, but typically not to the same extent or with such effects for plant growth. AM fungal hyphae also increase aggregation of soil particles through physical presence and production of binding agents, thereby altering soil structure (Rillig and Mummey 2006). AM fungi can improve plant water relations, particularly in arid environments, by water transport either within or along fungal hyphae (Augé 2001). Plants colonized by AM fungi can show enhanced 
pathogen protection (Borowicz 2001), through several potential mechanisms (Azcón-Aguilar and Barea 1997). Finally, some colonized plants exhibit increased tolerances to adverse environmental factors, such as herbivory (Gange and West 1994, Bennett and Bever 2007), heavy metals (Weissenhorn et al. 1994, Kaldorf et al. 1999), or salinity (Ruiz-Lozano et al. 1996). Although all these functions have been observed, it is not clear how often these different functions translate into observed benefits for plant hosts. It is also not clear if we have even discovered all potential functions of AM symbioses at this point.

Plant growth responses to AM fungi, usually characterized as mycorrhizal dependency or responsiveness, are a net indicator of all functions the fungus (or fungal community) provides and vary widely across fungi, plants, and environmental conditions (Johnson et al. 1997, Klironomos 2003, Smith and Read 2008). Most AM research has focused on a single function that fungi provide or quantified the total net effect of one fungal species/community on plant performance. By simultaneously examining the effect of different functions on plant responses, we can compare functional pathways among different $\mathrm{AM}$ fungi, plants and environments and establish when specific mycorrhizal functions are important to plant response. Furthermore, our approach provides critical information about the relative importance of non-estimated functions.

Here we estimate the relative importance of AM fungal functions on host plant biomass in two published data sets, one that measured multiple AM functions on a single plant species and another that measured a single AM function across multiple plant species. In these data sets, individual plants were grown in association with species of AM fungi under controlled environmental conditions. The AM fungal species used in these studies were selected as representatives of families that are hypothesized to be important for different types of functional interactions with plants (Maherali and Klironomos 2007, Powell et al. 2009, Sikes et al. 2009). Therefore, we were also able to estimate the relative importance of symbiont identity for the functions that were provided. We hypothesized that the relative importance of two mycorrhizal functions ( $\mathrm{P}$ uptake, pathogen protection) would vary based on the identity of the host species as well as the family-level affiliation of its fungal symbiont.

\section{Materials and Methods}

We used structural equation modeling to evaluate the relative contributions of estimated and non-estimated mycorrhizal functions to variation in host plant biomass. Structural equation modeling is a powerful tool to estimate direct and indirect relationships among dependent and independent variables by calculating partial regression and correlation coefficients (Wootton 1994). Models were specified and fit using functions in the sem package version 0.9-17 (Fox 2006) in R version
2.9.1 (R Core Development Team 2008). Standardized regression coefficients were estimated for paths leading from binary exogenous (i.e., not influenced by other variables in the model) variables associated with the presence or absence of AM fungi to an endogeneous dependent variable representing the aggregate outcome of mycorrhizal function for the host plant (biomass). Paths were specified via intervening endogenous variables representing mycorrhizal functions, including enhanced nutrient uptake and pathogen protection, and directly from the exogenous variables to the dependent variable, representing potential mechanisms of mycorrhizal functioning that were not estimated (hidden latent variables). Variables were log or arcsinesquare root transformed, when appropriate, to realize normality of error distributions and linear relationships among the variables.

The first data set contained estimates of total $\mathrm{P}$ in host plant tissue, host biomass production, and the frequencies of root fragments colonized by pathogenic fungi (Fusarium oxysporum, Pythium sp.) for 20 different AM fungal species associating with Plantago lanceolata in a greenhouse experiment (Maherali and Klironomos 2007). Host plants (one per pot) were grown for one year in sterilized soil inoculated with $50 \mathrm{~g}$ of roots $(P$. lanceolata) colonized by a single AM fungal species and with $1 \times 10^{6}$ spores of the pathogenic fungal species. Whole plant biomass was estimated from measurements of dried shoot and root tissue, foliar P was measured by spectrophotometry following dry ashing, and the extent of pathogen infection was estimated as a proportion of root length (Maherali and Klironomos 2007). For the purposes of the path model, AM fungal species were assigned to one of two binary dummy variables depending on their family-level affiliation (Glomeraceae, Gigasporaceae) since the mycorrhizal functions estimated here (reductions in pathogen infection, increased $\mathrm{P}$ uptake) appear to be strongly conserved within these two families (Powell et al. 2009). While there was no true nonmycorrhizal control in these data, we assume that plants inoculated with species in the Acaulosporaceae in this case represent a reasonable approximation; responses in mycorrhizal functions associated with taxa in this family did not differ significantly from those in other families that showed the least potential to influence these functions (Glomeraceae species for P content, Gigasporaceae species for pathogen reduction) in this experiment (Maherali and Klironomos 2007). We estimated the covariance matrix from the species-level means for each variable $(N=20)$, provided in the supplementary online materials from Maherali and Klironomos (2007).

The second data set contained estimates of host biomass production and the frequency of root fragments colonized by pathogenic fungi ( $F$. oxysporum) for six different AM fungal species, plus a nonmycorrhizal control treatment, associating with either Setaria glauca or Allium cepa in a greenhouse experiment (Sikes et al. 
2009). Host plants (one per pot) were grown for five months in sterilized soil containing one gram of roots (Allium porrum) colonized by a single AM fungal species. Each pot was then inoculated with $1 \times 10^{6}$ spores of $F$. oxysporum, then plants were harvested following a further four weeks of growth. Host plant biomass and percentage of the root length infected by the fungal pathogen were estimated as for the first data set (Sikes et al. 2009). As before, AM fungal species were assigned to binary dummy variables associated with family level affiliation. Separate models were fit for the two different host species in order to visualize the relative importance of mycorrhizal functions for hosts with different types of root architecture, which has been hypothesized as a major determinant of mycorrhizal functioning in the field (Newsham et al. 1995). For these data, the covariance matrix was estimated from all experimental units $(N=70)$ for each host plant species.

For each plant species, we used two approaches to evaluate the relative importance of paths in the model. First, we performed stepwise regression, via backward elimination, sequentially dropping paths from the fully parameterized model in order of ascending $z$-statistic (which represents the distance of the coefficient estimate from zero in units of standard error). Following each model fitting step, we calculated the likelihood ratio statistic to test the hypothesis that the elimination of a parameter does not result in a significant reduction in the fit of the model to the data. We identified the minimum adequate model as that which contained all parameters required to provide a good fit to the data based on the likelihood ratio test. As an alternative estimate of model fit, we also calculated the Akaike information criterion corrected for small sample size $\left(\mathrm{AIC}_{\mathrm{c}} ; \mathrm{McQuarrie}\right.$ and Tsai 1998) for each model. From the minimum adequate model, we determined the statistical significance (alpha $=0.05$ ) of paths from estimates of the unstandardized partial regression coefficients. We then estimated the total effect of AM fungi on plant biomass via the different functions (pathways in the model) as the sum of paths originating from each AM fungal variable (family). For a path connected by intermediate variables (indirect paths), we multiplied the partial standardized regression coefficients along the path to estimate the effect of the AM fungal variable on plant biomass. To graphically display the total effect associated with these pathways, we estimated the total effect on plant biomass predicted at a one standard deviation (SD) shift in the variables of interest $(\Delta \mathrm{SD}[x]=\Delta \mathrm{SD}$ [plant biomass] $\times$ [total effect], where $x$ represents variables on the pathway of interest; Bart and Earnst 1999).

Second, we estimated how including direct paths from plant $\mathrm{P}$, pathogen abundance, or AM fungal variables to plant biomass influenced model fit, starting with a model containing all paths except those leading directly to plant biomass. In addition, the importance of AM fungal family identity was determined by including paths leading from either one or both AM fungal variables to the relevant endogenous variable. In each case, we estimated four goodness-of-fit statistics: (1) the $\chi^{2}$ statistic (2 times the difference in log-likelihood of the fitted and fully parameterized model), a significantly high value of which indicates that the model fits the data poorly; (2) the Bayesian information criterion (BIC; Schwarz 1978), which invokes steeper penalties to models with more parameters; (3) the comparative fit index (CFI; Bentler 1990), (CFI > 0.97 indicates a good fit to data, 0.95-0.97 indicates an adequate fit to data; Schermelleh-Engel et al. 2003); and (4) $\mathrm{AIC}_{\mathrm{c}}$. We chose the latter two indices because they are appropriate for small sample sizes (McQuarrie and Tsai 1998, Schermelleh-Engel et al. 2003).

\section{RESUlts}

For all three of the host plant species for which we obtained data, we were able to partition variance in host biomass to at least one of the two AM fungal families. However, the functions associated with biomass variation differed among the three host species; this was indicated by the targeted inclusion of variables associated with specific functions (Table 1) and by the strength of standardized partial regression coefficients in the minimum adequate models (Figs. 1, 2).

Increased biomass of $P$. lanceolata was strongly associated with enhanced $\mathrm{P}$ uptake by AM fungal species in the Gigasporaceae (Table 1, Fig. 1). Significant negative associations were observed for paths from AM fungal species in the Glomeraceae to the estimates of pathogen infection frequency, although these were inconsistently associated with variation in host biomass (negative for the Pythium sp., positive for F. oxysporum). The predicted effect of AM fungi in these two families on plant biomass was weak for paths associated with pathogen protection compared to that of the path associated with nutrient uptake (Fig. 1). Model selection based on likelihood ratio statistics suggested a minimum adequate model that included paths from both pathogen variables to plant biomass (model summary presented in Table 1), while the $\mathrm{AIC}_{\mathrm{c}}$ index suggested a minimum adequate model that excluded these paths. For the model that included these paths, effects associated with AM fungal-mediated nutrient uptake contributed to $53 \%$ of the total effect predicted by the model, while the contribution of Pythium sp. and F. oxysporum infection was $25 \%$ and $22 \%$, respectively.

For the other two host species, increases in host biomass were significantly associated with paths passing directly from the AM fungal variables, suggesting that this variation was not accounted for by the frequency of pathogen infection in roots. For A. cepa, coefficients leading from both AM fungal variables were significantly positive, with the Gigasporaceae variable indicating a stronger association (Fig. 2); paths originating from both AM fungal variables were necessary to ensure adequate model fit (Table 1). For S. glauca, only species

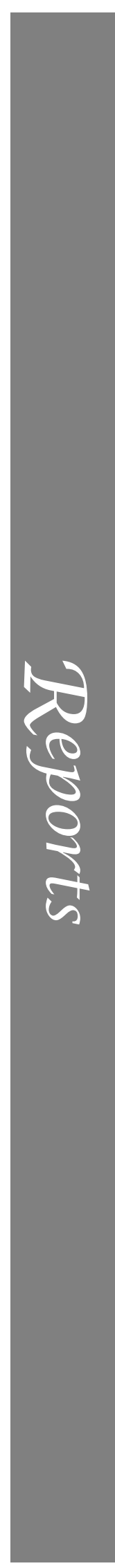


TABLE 1. Comparison of structural equation models for the estimation of arbuscular mycorrhizal fungal effects on host plant biomass via different symbiotic functions (paths).

\begin{tabular}{|c|c|c|c|c|}
\hline Model† & Chi-square & CFI & $\mathrm{BIC} \S$ & $\mathrm{AIC}_{\mathrm{c}} \boldsymbol{\top}$ \\
\hline \multicolumn{5}{|l|}{ Host Plantago lanceolata } \\
\hline $\begin{array}{l}\text { No paths to host } \\
\text { + Direct paths }\end{array}$ & $37.48(5)^{* * *}$ & 0.664 & 22.50 & 124.14 \\
\hline Via AM & $26.01(3)^{* * *}$ & 0.762 & 17.02 & 176.01 \\
\hline Via Glo & $35.89(4)^{* * *}$ & 0.670 & 23.91 & 147.89 \\
\hline Via Gig & $27.31(4)^{* * *}$ & 0.759 & 15.32 & 139.31 \\
\hline \multicolumn{5}{|l|}{+ Phosphorus paths } \\
\hline Via AM & $7.83(4)$ & 0.960 & -4.15 & 119.83 \\
\hline Via Glo & $19.78(5)^{* *}$ & 0.847 & 4.80 & 106.45 \\
\hline Via Gig & $8.25(5)$ & 0.966 & -6.73 & 94.91 \\
\hline \multicolumn{5}{|l|}{+ Pathogen paths } \\
\hline Via AM & $44.12(5)^{* * *}$ & 0.595 & 29.14 & 130.78 \\
\hline Via Glo & $44.80(7)^{* * *}$ & 0.608 & 23.83 & 99.80 \\
\hline Via Gig & $68.60(7)^{* * *}$ & 0.362 & 47.63 & 123.60 \\
\hline Min. adequate (Fig. 1) & $4.37(8)$ & 1.000 & -19.60 & 48.81 \\
\hline \multicolumn{5}{|l|}{ Host Setaria glauca } \\
\hline $\begin{array}{l}\text { No paths to host } \\
\text { + Direct paths }\end{array}$ & $69.28(3)^{* * *}$ & 0.660 & 56.53 & 77.89 \\
\hline Via AM & $1.82(1)$ & 0.996 & -2.43 & 15.15 \\
\hline Via Glo & $3.07(2)$ & 0.994 & -5.42 & 14.01 \\
\hline Via Gig & $46.11(2)^{* * *}$ & 0.774 & 37.61 & 57.05 \\
\hline \multicolumn{5}{|l|}{ + Pathogen paths } \\
\hline Via AM & $23.65(2)^{* * *}$ & 0.889 & 15.15 & 34.58 \\
\hline Via Glo & $23.67(3)^{* * *}$ & 0.894 & 10.92 & 32.28 \\
\hline Via Gig & $65.43(3)^{* * *}$ & 0.680 & 52.69 & 74.05 \\
\hline Min. adequate (Fig. 2) & 3.09 (3) & 1.000 & -9.65 & 11.71 \\
\hline \multicolumn{5}{|l|}{ Host Allium cepa } \\
\hline $\begin{array}{l}\text { No paths to host } \\
+ \text { Direct paths }\end{array}$ & $38.00(3)^{* * *}$ & 0.609 & 25.26 & 46.62 \\
\hline Via AM & $0.12(1)$ & 1.000 & -4.13 & 13.45 \\
\hline Via Glo & $32.00(2)^{* * *}$ & 0.665 & 23.51 & 42.94 \\
\hline Via Gig & $6.99(2)^{*}$ & 0.944 & -1.50 & 17.93 \\
\hline \multicolumn{5}{|l|}{ + Pathogen paths } \\
\hline Via AM & $37.99(2)^{* * *}$ & 0.598 & 29.49 & 48.93 \\
\hline Via Glo & $38.09(3)^{* * *}$ & 0.608 & 25.34 & 46.70 \\
\hline Via Gig & $38.02(3)^{* * *}$ & 0.609 & 25.28 & 46.64 \\
\hline Min. adequate (Fig. 2) & $0.60(3)$ & 1.000 & -12.14 & 9.22 \\
\hline
\end{tabular}

Notes: The chi-square value is a likelihood-ratio chi-square statistic between the chi-square statistics of the saturated model (with all pathways) and the model with the individual pathway elements. It represents the difference in fit to the data between the two models, with low values indicating more similar fits between models. The chi-square statistic for each model is an estimate of residual deviance of the model covariance matrix from the data matrix. Degrees of freedom are given in parentheses. Significant tests (indicated with asterisks) indicate that the model matches data poorly.

$* P<0.05 ; * * P<0.01 ; * * * P<0.001$.

+ SEM model containing specified paths to plant biomass, with scores indicating good or adequate fit in bold; paths originated from either (Glo, Glomeraceae species; Gig, Gigasporaceae species) or both (AM) of the fungal variables. The minimum (min.) adequate model contains the fewest paths, following stepwise regression, necessary to maintain a good fit between the model and data covariance matrices.

t Comparative fit index (Bentler 1990): $>0.97$, good fit to data; 0.95-0.97, adequate fit to data (Schermelleh-Engel et al. 2003).

$\S$ Bayesian information criterion (Schwarz 1978); model with the lowest value has the best fit to the data.

- Akaike information criterion corrected for small sample size (McQuarrie and Tsai 1998); model with the lowest value has the best fit to the data.

within the Glomeraceae enhanced plant biomass (Fig. 2 ); the model containing only this path fit the data better than the model containing direct paths from both AM fungal variables (Table 1). This biomass promotion may have been associated with pathogen protection, but this was apparently not related to reductions in the frequency of root infection by the pathogen: the coefficient associated with this path was not significant 


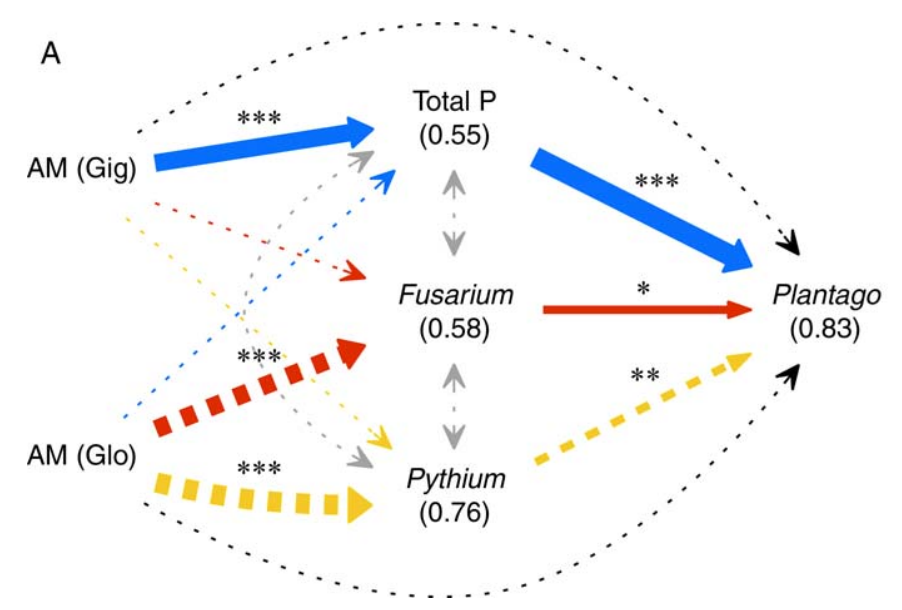

\section{B}

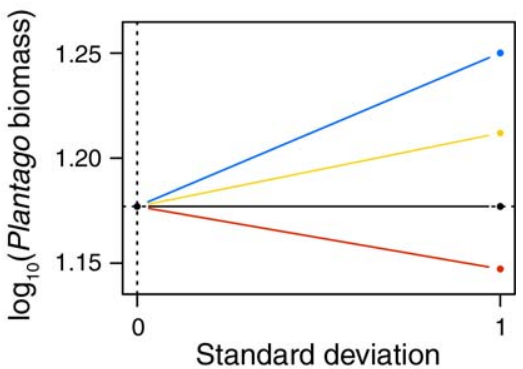

FIG. 1. Relative importance of arbuscular mycorrhizal (AM) fungal-mediated phosphorus (P) uptake and reduction in pathogenic fungal infection (Fusarium oxysporum, Pythium sp.) on biomass of Plantago lanceolata. (A) Structural equation model (SEM) showing the effect of observed and hidden latent variables on plant biomass. Directional effects and correlations between pairs of variables are represented by single- and double-headed arrows, respectively; solid and dashed arrows represent positive and negative relationships, respectively. Asterisks indicate statistically significant associations; dotted arrows are nonsignificant paths that were dropped from the fully parameterized model. Arrow widths for the significant paths correspond to the effect size, determined by the standardized partial regression coefficient ( $\beta$; for scale, $\beta_{\text {Fusarium } \Rightarrow \text { Plantago }}=0.353, \beta_{\text {Total }} \Rightarrow$ Plantago $=0.888$ ). AM fungal taxa were grouped by family affiliation (Glo, Glomeraceae; Gig, Gigasporaceae). Numbers in parentheses correspond to the proportion of variance explained by the model $\left(R^{2}\right)$ for each variable. (B) The predicted effect of AM fungi (Glomeraceae and Gigasporaceae combined) on plant biomass based on the minimum adequate SEM. Effect on biomass was determined using the equation $\Delta \mathrm{SD}(x)=\Delta \mathrm{SD}$ (biomass) $\times \mathrm{TE}$, where $x$ is a path from AM fungal variables to plant biomass, via total $\mathrm{P}$ (blue), Fusarium (red), Pythium (yellow), or latent variables (black), and TE is the total effect calculated from the SEM (see Methods). At 0 SD of $x$, we used the mean value of plant biomass (log-transformed) over all AM fungal treatments $\left(10^{1.18} \mathrm{~g}\right)$.

$* P<0.05 ; * * P<0.01 ; * * * P<0.001$.

even though species within this family were associated with a strong reduction in pathogen infection.

\section{Discussion}

Utilizing this approach, we successfully quantified the relative importance of different AM fungal functions to changes in plant biomass. Within the multifunctional model we could discern that enhanced phosphorus uptake associated with AM fungal species in the Gigasporaceae increased growth of $P$. lanceolata to a greater extent than effects on pathogen abundance by species of Glomus (Fig. 1). When only one function was measured, the relative importance of this function was dependent on the plant species (Fig. 2). As expected, we observed no association between pathogen protection and growth of A. cepa, a host with a poorly-branched root system. Plants with limited root surface area are hypothesized to be less susceptible to pathogen infection and more likely to benefit from enhanced nutrient and water uptake by mycorrhizas (Newsham et al. 1995, Sikes et al. 2009), functions that are likely represented by the direct paths in our model (particularly from species in the Gigasporaceae). In contrast, S. glauca possesses a highly branched root system, generally associated with efficient foraging ability but hypothesized to be highly susceptible to pathogen infection (Newsham et al. 1995, Sikes et al. 2009). Glomus species significantly reduced the frequency of infection by F. oxysporum in this host; however this functional pathway was not an important predictor of plant biomass. Glomus species increased biomass of $S$. glauca via unmeasured functions (Fig. 2: direct pathway) that fully compensated for $F$. oxysporum-induced reductions in biomass of non-mycorrhizal S. glauca (Sikes et al. 2009), suggesting that this effect is associated, in some way, with pathogen protection by AM fungi. We also observed a weak, positive correlation between $F$. oxysporum infection frequency and P. lanceolata biomass (Fig. 1), which further suggests the association between these variables is more complicated than strictly pathogenic.

Choosing appropriate metrics for quantifying functions is critical to defining their relative importance. For example, the frequency of infected root segments may be a suitable predictor of host biomass for some pathogens (such as for Pythium in Fig. 1); however, measurement of alternative traits associated with pathogen protection may be necessary to better quantify the relative importance of this function. AM fungal-mediated pathogen protection may be due to several different mechanisms (Azcón-Aguilar and Barea 1997, Whipps 2004), and choosing the appropriate mechanism to quantify and a suitable trait associated with the mechanism are not trivial concerns. One of the advantages of structural equation modeling is that multiple traits can be combined to form latent variables, which are not directly observed but are inferred from measured variables (Bentler 1980). This procedure will be useful where estimation of 
A

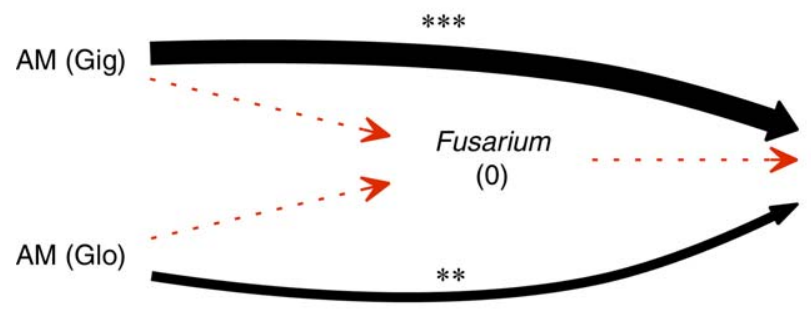

C

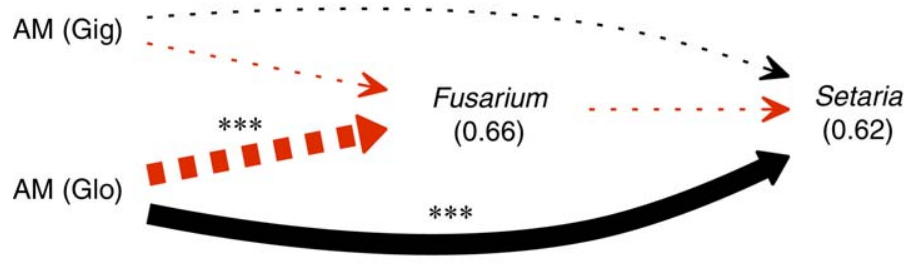

B

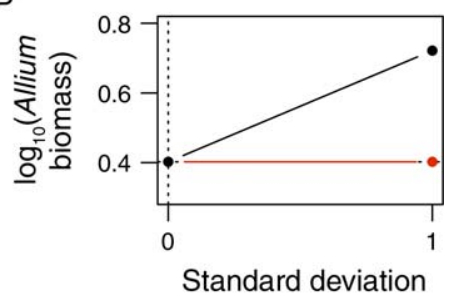

D

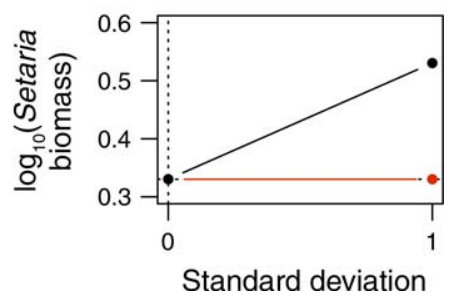

FIG. 2. Relative importance of arbuscular mycorrhizal (AM) fungal-mediated reduction in pathogenic fungal infection (Fusarium oxysporum) on biomass of (A, B) Allium cepa and (C, D) Setaria glauca. (A, C) Structural equation model (SEM) showing the effect of observed and hidden latent variables on plant biomass. Directional effects and correlations between pairs of variables are represented by single- and double-headed arrows, respectively; solid and dashed arrows represent positive and negative relationships, respectively. Asterisks indicate statistically significant associations; dotted arrows are nonsignificant paths that were dropped from the fully parameterized model. Arrow widths for the significant paths correspond to the effect size, determined by the standardized partial regression coefficient $\left(\beta\right.$; for scale, $\left.\beta_{\mathrm{AM}(\mathrm{Glo}) \Rightarrow \text { Allium }}=0.372, \beta_{\mathrm{AM}(\mathrm{Gig}) \Rightarrow \text { Allium }}=0.881\right)$. AM fungal taxa were grouped by family affiliation (Glo, Glomeraceae; Gig, Gigasporaceae). Numbers in parentheses correspond to the proportion of variance explained by the model $\left(R^{2}\right)$ for each variable. (B, D) The predicted effect of AM fungi (Glomeraceae and Gigasporaceae combined) on plant biomass based on the minimum adequate SEM. Effect on biomass was determined using the equation $\Delta \mathrm{SD}(x)=\Delta \mathrm{SD}$ (biomass) $\times \mathrm{TE}$, where $x$ is a path from $\mathrm{AM}$ fungal variables to plant biomass, via Fusarium (red) or latent variables (black), and TE is the total effect calculated from the SEM (see Methods). At 0 SD of $x$, we used the mean value of plant biomass (log-transformed) over all AM fungal treatments (for Allium, $10^{0.40} \mathrm{~g}$; for Setaria, $10^{0.33} \mathrm{~g}$ ).

** $P<0.01 ; * * *<0.001$.

individual traits may not be sufficient to adequately represent the overall effect of these pathways.

In addition, this approach could effectively deal with higher degrees of functional complexity than we addressed here. For instance, plant biomass could be evaluated in the presence and absence of AM fungi by estimating several variables associated with nutrient uptake (including $\mathrm{P}, \mathrm{N}$, and various micronutrients), pathogen protection (pathogen abundance, metabolic efficiency of infected hosts), and soil stability (frequency of water-stable aggregates, aggregate diameter). Such responses could be estimated following the inoculation of plants with AM fungal isolates or assemblages under controlled greenhouse conditions, as was done here, or by comparing responses under natural conditions to those following the experimental elimination of $\mathrm{AM}$ fungi (Wilson et al. 2009). The latter approach would be especially valuable to further understand the context dependence of different symbiotic functions and their importance to plant productivity and community structure. For example, if estimates of mycorrhizal dependency were collected over gradients in nutrient availability, pathogen abundance, and soil texture, variance in plant response could then be partitioned among paths associated with these variables. In a community context, this framework could distinguish whether the influence of AM fungal diversity on plant diversity and productivity (van der Heijden et al. 1998) is a product of AM functional complementarity or due to an increased likelihood of plants associating with a preferred fungus for a single function. This knowledge can then be used to help land managers tailor inoculants to provide important functions that promote specific plants in agriculture or ecosystem restoration.

Application of this framework can also effectively delineate multifunctionality in other plant-microbial symbioses, provided the data are available. Symbioses that vary along specific genotypes (grass endophytes, Rhizobia) or entire functional groups (ectomycorrhizal fungi) can establish the relative importance of functions we are measuring and those that we are not. Many of these microbial symbioses have almost no information about multiple functions and utilizing measurements of several functions within this framework can rapidly screen for potentially important pathways. An essential component to utilize this framework for both known and yet-to-be discovered microbial symbioses is to establish data that simultaneously capture multiple functions. Although multifunctionality adds another layer to an already complex group of interactions, 
utilizing this approach can differentiate mechanistic pathways in complex plant-microbial symbioses and provide a common template to compare them.

\section{ACKNOWLEDGMENTS}

B. A. Sikes and J. R. Powell contributed equally to the publication of this work. We thank Tancredi Caruso, Ryan Norris, Matthew Bowker, John Klironomos, and one anonymous reviewer for advice and comments on the manuscript. We also thank the staff of the Luise Biergarten for delaying the arrival of our drinks which allowed proper stratification and germination of this project. This research was supported by a Center for International Cooperation grant through the Freie Universität Berlin to B. A. Sikes and a Marie Curie Postdoctoral Grant to J. R. Powell.

\section{Literature Cited}

Arora, N. K., S. C. Kang, and D. K. Maheshwari. 2001. Isolation of siderophore-producing strains of Rhizobium meliloti and their biocontrol potential against Macrophomina phaseolina that causes charcoal rot of groundnut. Current Science 81:673-677.

Augé, R. M. 2001. Water relations, drought and vesiculararbuscular mycorrhizal symbiosis. Mycorrhiza 11:3-42.

Azcón-Aguilar, C., and J. M. Barea. 1997. Arbuscular mycorrhizas and biological control of soil-borne plant pathogens: an overview of the mechanisms involved. Mycorrhiza 6:457-464.

Bart, J., and S. L. Earnst. 1999. Relative importance of male and territory quality in pairing success of male rock ptarmigan (Lagopus mutus). Behavioral Ecology and Sociobiology 45:355-359.

Bennett, A. E., and J. D. Bever. 2007. Mycorrhizal species differentially alter plant growth and response to herbivory. Ecology 88:210-218.

Bentler, P. M. 1980. Multivariate analysis with latent variables: causal modeling. Annual Review of Psychology 31:419-456.

Bentler, P. M. 1990. Comparative fit indexes in structural models. Psychological Bulletin 107:238-246.

Bolan, N. S. 1991. A critical review on the role of mycorrhizal fungi in the uptake of phosphorus by plants. Plant and Soil 134:189-207.

Borowicz, V. A. 2001. Do arbuscular mycorrhizal fungi alter plant-pathogen relations? Ecology 82:3057-3068.

Chaudhary, V., M. Bowker, T. O'Dell, J. Grace, A. Redman, M. C. Rillig, and N. C. Johnson. 2009. Untangling the biological contributions to soil stability in semiarid shrublands. Ecological Applications 19:110-122.

Finlay, R. D. 2008. Ecological aspects of mycorrhizal symbiosis: with special emphasis on the functional diversity of interactions involving the extraradical mycelium. Journal of Experimental Botany 59:1115.

Fox, J. 2006. Structural equation modeling with the sem package in R. Structural Equation Modeling 13:465-486.

Gange, A. C., and H. M. West. 1994. Interactions between arbuscular mycorrhizal fungi and foliar-feeding insects in Plantago lanceolata L. New Phytologist 128:79-87.

Johnson, N. C., J. H. Graham, and F. A. Smith. 1997. Functioning of mycorrhizal associations along the mutualism-parasitism continuum. New Phytologist 135:575-586.

Kaldorf, M., A. J. Kuhn, W. H. Schröder, U. Hildebrandt, and H. Bothe. 1999. Selective element deposits in maize colonized by a heavy metal tolerance conferring arbuscular mycorrhizal fungus. Journal of Plant Physiology 154:718-728.

Klironomos, J. N. 2003. Variation in plant response to native and exotic arbuscular mycorrhizal fungi. Ecology 84:22922301

Maherali, H., and J. N. Klironomos. 2007. Influence of phylogeny on fungal community assembly and ecosystem functioning. Science 316:1746-1748.

Mandyam, K., and A. Jumpponen. 2005. Seeking the elusive function of the root-colonising dark septate endophytic fungi. Studies in Mycology 53:173.

McQuarrie, A. D. R., and C. Tsai. 1998. Regression and time series model selection. World Scientific, Hackensack, New Jersey, USA.

Newsham, K. K., A. H. Fitter, and A. R. Watkinson. 1995 Multi-functionality and biodiversity in arbuscular mycorrhizas. Trends in Ecology and Evolution 10:407-411.

Powell, J. R., J. Parrent, M. M. Hart, J. N. Klironomos, M. C. Rillig, and H. Maherali. 2009. Phylogenetic trait conservatism and the evolution of functional trade-offs in arbuscular mycorrhizal fungi. Proceedings of the Royal Society B 276: $4237-4235$.

R Core Development Team. 2008. R: a language and environment for statistical computing. R Project for Statistical Computing, Vienna, Austria. 〈http://www.R-project. org $\rangle$

Rillig, M. C., and D. L. Mummey. 2006. Mycorrhizas and soil structure. New Phytologist 171:41-53.

Ruiz-Lozano, J. M., R. Azcon, and M. Gomez. 1996. Alleviation of salt stress by arbuscular-mycorrhizal Glomus species in Lactuca sativa plants. Physiologia Plantarum 98: 767-772.

Schardl, C. L., A. Leuchtmann, and M. J. Spiering. 2004 Symbioses of grasses with seedborne fungal endophytes. Annual Review of Plant Biology 55:315-340.

Schermelleh-Engel, K., H. Moosbrugger, and H. Müller. 2003. Evaluating the fit of structural equation models: tests of significance and descriptive goodness-of-fit measures. Methods of Psychological Research 8:23-74.

Schwarz, G. 1978. Estimating the dimension of a model. Annals of Statistics 6:461-464.

Sikes, B. A., K. Cottenie, and J. N. Klironomos. 2009. Plant and fungal identity determines pathogen protection of plant roots by arbuscular mycorrhizas. Journal of Ecology 97: $1274-1280$.

Smith, S. E., and D. J. Read. 2008. Mycorrhizal symbiosis. Academic Press, San Diego, California, USA.

van der Heijden, M. G., J. N. Klironomos, M. Ursic, P. Moutoglis, R. Streitwolf-Engel, T. Boller, A. Wiemken, and I. R. Sanders. 1998. Mycorrhizal fungal diversity determines plant biodiversity, ecosystem variability and productivity. Nature 100:912-927.

Vázquez, D. P., and D. Simberloff. 2004. Indirect effects of an introduced ungulate on pollination and plant reproduction. Ecological Monographs 74:281-308.

Weissenhorn, I., A. Glashoff, C. Leyval, and J. Berthelin. 1994. Differential tolerance to $\mathrm{Cd}$ and $\mathrm{Zn}$ of arbuscular mycorrhizal (AM) fungal spores isolated from heavy metal-polluted and unpolluted soils. Plant and Soil 167:189-196.

Whipps, J. M. 2004. Prospects and limitations for mycorrhizas in biocontrol of root pathogens. Botany 82:1198-1227.

Wilson, G., C. Rice, M. Rillig, A. Springer, and D. Hartnett. 2009. Soil aggregation and carbon sequestration are tightly correlated with the abundance of arbuscular mycorrhizal fungi: results from long-term field experiments. Ecology Letters 12:452-461.

Wootton, J. 1994. Predicting direct and indirect effects: an integrated approach using experiments and path analysis. Ecology 75:151-165. 